

www.laender-analysen.de/polen

\title{
POLEN UND LITAUEN - EINE KOMPLIZIERTE NACHBARSCHAFT
}

ANALYSE

Polen und Litauen - eine komplizierte Nachbarschaft

Michał Olszewski, Krakau

DOKUMENTATION

Außenminister Sikorski und Außenminister Linkevičius über die polnisch-litauische

Zusammenarbeit

Außenministerium: Neue Dynamik in den Beziehungen zu Litauen nach der

Verabschiedung des Namensgesetzes

Sikorski: Gaspipeline in die baltischen Länder gegen die Isolation der Region

- UMFRAGE

Sympathie gegenüber anderen Nationen

CHRONIK

Vom 5. bis zum 18. März 2013

Die nächste Ausgabe der Polen-Analysen erscheint nach der Osterpause am 16. April 2013.

Herausgegeben mit finanzieller Unterstützung der Stiftung für deutsch-polnische Zusammenarbeit
FUNDACJA WSPÓŁPRACY

POLSKO-NIEMIECKIEJ

STIFTUNG

FÜR DEUTSCH-POLNISCHE

ZUSAMMENARBEIT
DEUTSCHES POLEN INSTITUT 


\title{
Polen und Litauen - eine komplizierte Nachbarschaft
}

\author{
Michał Olszewski, Krakau
}

\section{Zusammenfassung}

Auch wenn man eine kurze politische Analyse nicht mit persönlichen Bekenntnissen beginnen sollte, sind sie hier doch notwendig. In den vergangenen Jahren habe ich mich bemüht, die immer angespannteren Beziehungen zwischen Polen und Litauen zu verfolgen - sowohl aus der entfernten Krakauer Perspektive als auch von Orten aus, die bedeutend näher an Wilna liegen. Aufgewachsen in Masuren, also gewissermaßen in der Nähe der litauischen Hauptstadt, in einer Familie, die seit Generationen im polnisch-litauischen Grenzgebiet ansässig ist, kam ich zu der Überzeugung, dass die Eskalation der gegenseitigen Vorwürfe ausschließlich durch die Ängste und Ressentiments der litauischen Seite hervorgerufen wurde. Die einzig wirksame Antwort auf die evidente Missachtung der Rechte der polnischen Minderheit in Litauen könne daher nur eine harte, kompromisslose Politik Warschaus sein. Die vergangenen Monate erlauben mir, die Vernünftigkeit dieser Forderungen zu überprüfen, denn sie haben sich als naiv erwiesen. Auf Initiative von Außenminister Radosław Sikorski begann die polnische Seite eine Offensive, die bis auf weiteres allerdings ohne diplomatischen Charme geführt wird, auf Ungeduld beruht und darauf, den "widerspenstigen« jüngeren Bruder zurechtzuweisen.

$\mathrm{B}^{\mathrm{i}}$ is zu einem gewissen Grad scheint die Irritation der Regierung von Ministerpräsident Donald Tusk verständlich zu sein. Im komplizierten Geflecht der polnischen politischen Konflikte nach 1989 waren nämlich gutnachbarschaftliche Beziehungen zu Litauen immer eines der wenigen Dogmen der unterschiedlichen Regierungen. Die historisch begründeten Ängste unseres Nachbarn wurden als verständlich bewertet, umso mehr, als uns bis zum Jahr 2004 gemeinsame Bemühungen verbanden - zunächst der Beitritt zur NATO, dann zur EU und schließlich zum Schengen-Abkommen. Diese Strategie hat ihre Prüfung bestanden. Ein sichtbares Zeichen ihres Erfolgs ist beispielsweise der Grenzübergang in Budzisk (Woiwodschaft Podlachien). Noch vor nicht allzu langer Zeit war dies ein gefürchteter Ort, der von mafiösen Strukturen beherrscht wurde, mit kilometerlangen LKW-Schlangen, die, so schien es, dort bis zum Weltende stehen würden. Aber sie sind verschwunden.

Eine arrogante Politik Litauen gegenüber zu vermeiden, hatte in der III. Republik also einen pragmatischen Grund. Zugegeben werden muss auch, dass der Neuordnung der Beziehungen ein fundamentaler Missklang zugrunde lag, über den verlegen geschwiegen werden musste. Ich denke hier an die Unterstützung, die ein Teil der litauischen Polen Gennadi Janajew beim Putsch gegen Michail Gorbatschow am 19. August 1991 gewährt hatte, weil diese litauischen Polen darin eine Chance für die frisch ausgerufene autonome Region innerhalb Litauens sahen.

\section{Schwierige Rahmenbedingungen für eine gute Nachbarschaft}

Die geopolitischen Erfolge hatten jedoch einen hohen Preis. Auf ihrem Altar wurden vor allem die polnischen
Litauer geopfert. Krönender Beweis ist das litauische Reprivatisierungsgesetz von 1997. Die Polen wurden ganz ans Ende der Warteschlange für Entschädigungen gedrängt. Familien aus Wilna beispielsweise warteten trotz eingereichter vollständiger Dokumente etliche Jahre auf die Entscheidung der Behörden und beobachteten dabei, wie die von ihnen reservierten Parzellen in den Besitz von Litauern übergingen. Berücksichtigt man dabei noch die Tatsache, dass das Gesetz es ermöglichte, die Entschädigung für das von der UdSSR enteignete Land an einem beliebigen Ort einzulösen, was zu Veränderungen im Gebiet um die Hauptstadt und zu einer Verdünnung des polnischen Elements im Wilnaer Gebiet führte, erhält man ein Bild von der offenkundigen Ungerechtigkeit, auf die Warschau nicht reagierte.

Hinzu kommen Probleme des Bildungswesens. Im leerer werdenden Land bedarf es einer Bildungsreform ähnlich derjenigen, die in Polen durchgeführt worden ist. In beiden Ländern werden die Dörfer verlassen, es bleiben dort die Alten, die Erfolglosen sowie die Besitzer landwirtschaftlicher Großbetriebe. Damit geht einher, dass die Ausbildung in diesen Gebieten für den Staat und die lokalen Behörden eine große Belastung darstellt. Allerdings weckt die Art und Weise, wie die Litauer das Bildungswesen reformieren, Zweifel. Die Verringerung der Anzahl der Schulfächer, die in der Muttersprache gelehrt werden (der Streit betrifft nicht nur Polnisch als Unterrichtssprache), oder auch die Vereinheitlichung des Abiturs im Schulfach Litauisch weckten eine Reihe von Protesten unter den Schülern der litauischen Schulen sowie denen der Minderheit. Die Erklärung, dass die Änderungen der Sorge um die polnischen Kinder und deren zukünftige Konkurrenzfähigkeit auf dem Arbeitsmarkt entspringen, klingt nicht überzeugend. Im 
Gegensatz zu der mit der polnischen und der russischen Sprache aufgewachsenen Elterngeneration spricht die jüngste Schülergeneration ganz natürlich Polnisch und Litauisch. Angaben der polnischen Minderheit zufolge werden 70 Prozent der jungen Polen in Studienfächer, die auf Litauisch gelehrt werden, aufgenommen.

\section{Streit über die Schreibweise von Nachnamen} Bitterkeit rief auch die Frage der Schreibweise von Nachnamen hervor. In Litauen wird um jedes diakritische Zeichen gekämpft. Der polnisch-litauische Vertrag von 1994 präzisiert, dass sowohl die Polen in Litauen als auch die Litauer, die in Polen leben, das Recht haben, ihre Namen in der Weise zu schreiben, die sie für richtig halten. Trotzdem bleiben die polnischen Namen in Litauen nach wie vor lituanisiert. Der Spruch des litauischen Verfassungsgerichts im Herbst 2009 goss noch Öl ins Feuer. Das Gericht entschied, dass die litauischen Bürger mit einer anderen Nationalität als der litauischen die Pflicht haben, ihre Namen in litauischer Schreibweise auf den ersten Seiten von Dokumenten anzugeben. Die Schreibweise in einer anderen Sprache soll nur eine Hilfe sein und kann auf den folgenden Seiten des Dokuments verwendet werden. Die litauische Regierung verwies darauf, dass diese Entscheidung im Widerspruch zu den Empfehlungen des Europäischen Gerichtshofs für Menschenrechte steht und die bürgerlichen Freiheiten vergewaltigt. Als Antwort darauf lehnte das von den Konservativen dominierte Parlament den Regierungsentwurf des Gesetzes ab. Die Polen in Litauen haben diese Schlacht verloren.

Die Absurdität dieser Situation kann man auf Schritt und Tritt sehen. Der Bürgermeister von Šalčininkai (südlich von Wilna, poln.: Soleczniki), dessen Einwohner mehrheitlich Polen sind, hat, obwohl er Pole ist, theoretisch die Pflicht, mit Gästen aus Polen und polnischen Antragstellern ausschließlich in der Amtssprache Litauisch zu sprechen. Um die Erlaubnis, dass Vor- und Nachname von Adam Mickiewicz auf einem Denkmal auf Polnisch geschrieben werden durften, fochten die Behörden einen regelrechten Kampf aus und wandten schließlich eine List an: Angebracht wurde eine Vergrößerung der Unterschrift von Mickiewicz. Nun können sie erklären, dass es sich auf dem Denkmal um ein Faksimile handelt und nicht um den amtlichen litauischen Namen des Dichters. Diejenigen, die wollen, dass Namen in der von der Minderheit vorgeschlagenen Version geschrieben werden können, gehörten zum Abschaum der Gesellschaft und strebten die »Slawisierung« Litauens an - ist zu hören. "Slawisierung« ist ein Ausdruck, mit dem die litauischen Konservativen gern die Wähler erschrecken, wobei sie vor allem das Bild eines räuberischen Polen entwerfen, das es auf die Souveränität des nördlichen Nachbarn abgesehen hat.

\section{Missglückte Versuche einer wirtschaftlichen und energiepolitischen Partnerschaft}

Mit einer unverständlichen Niederlage endeten auch die bisherigen Versuche, eine wirtschaftliche Partnerschaft aufzubauen. Das Lieblingskind von Staatspräsident Lech Kaczyński, der die politischen und wirtschaftlichen Beziehungen zwischen Warschau und Wilna zu stärken versuchte, war die Investition des polnischen Erdölkonzerns PKN Orlen in Mozejki. Die Erdölraffinerie war jahrelang ein Verlustgeschäft und ihr drohte die Schließung. Hilfserklärungen stießen brutal mit der Realität zusammen. Aus ungeklärten Ursachen montierten die litauischen Eisenbahnen gleich, nachdem die Russen die Rohstofflieferung zu der von den Polen übernommenen Raffinerie gestoppt hatten, die Schienenanlagen ab, die einen schnelleren und billigeren Erdöltransport zur Raffinerie ermöglichten. (WikiLeaks deckte auf, dass Russland absichtlich eine Havarie der Pipeline verursacht hatte, um einen Zusammenbruch des Unternehmens herbeizuführen.) Nach jahrelangen Überlegungen entschied Orlen, dass es die Anlage in Mozejki trotz allem nicht verkaufen wird, aber Bitterkeit und Misstrauen blieben auf beiden Seiten bestehen. Möglicherweise betrachteten die Litauer die Übernahme der Raffinerie als Symbol des polnischen Großmachtstrebens.

Ein symbolischer Moment des Zusammenbruchs der polnisch-litauischen Beziehungen war der 8. April 2010, zwei Tage, bevor das Flugzeugunglück von Smolensk geschah. Die damalige parlamentarische Mehrheit in Litauen erteilte Lech Kaczyński während seines letzten Besuches vor seinem tödlichen Unfall in Litauen eine heftige Ohrfeige, indem sie das für die Minderheit ungünstige Gesetz über die Schreibweise von Namen verabschiedete. Diese Geste wurde für kurze Zeit von der nationalen Tragödie Polens verdrängt, sie tauchte aber wieder auf, als die Emotionen über das Unglück abgeklungen waren. Der Streit dauert bis heute an; in Litauen wird er nahezu als Schlacht um die Unabhängigkeit behandelt.

\section{Suche nach neuen strategischen Partnerschaften in der Region}

Das Hauptelement der neuen polnischen Strategie in diesem Teil Europas ist der Aufbau einer strategischen Partnerschaft mit Lettland und Estland, Länder, die Außenminister Sikorski als attraktive Vervollständigung der Visegrád-Gruppe betrachtet. Der Besuch von Ministerpräsident Donald Tusk in Tallinn (Estland) am 11. März 2013 war die Krönung der mehrmonatigen Maßnahmen und Konsultationen auf Ministerebene. Für Polen kann die Stärkung der Position im ehemaligen sowjetischen "Pribaltikum» (Litauen, Lettland, Estland) ein stärkeres Mandat für die Rolle als Führer 
in der Region bedeuten. Die bislang hinkende Zusammenarbeit dieser EU-Mitgliedsländer, die vor nicht allzu langer Zeit noch im sowjetischen Einflussbereich lagen, kann an Schwung gewinnen, was für Polen auf der Suche nach Verbündeten zumindest im Kampf gegen die EUKlimapolitik eine ungeheure Bedeutung hätte. Für Lettland und Estland wiederum ist Polen das Fenster zum Westen. Eine gemeinsame Front kann zum Beispiel eine leichtere Realisierung großer Transportprojekte bedeuten. $\mathrm{Zu}$ nennen wäre hier die seit Jahren von den Ländern der Region erwartete Eisenbahntrasse Rail Baltica von Warschau nach Helsinki, deren Umsetzung im Frühjahr 2013 beginnen soll. Die Ambitionen der EU zielen noch weiter. Angestrebt ist die Schaffung einer starken baltischen Makroregion, die in der Lage sein soll, mit der in dieser Region immer aktiver werdenden Russischen Föderation zu konkurrieren.

Wo ist hier ein Platz für Litauen? Obgleich es flächenmäßig klein ist (ein Fünftel der Fläche Polens) und sich erheblichen demographischen Problemen stellen muss (vorsichtigen Schätzungen zufolge emigrierten in den letzten 20 Jahren 500.000 Personen von 3,5 Millionen Einwohnern dauerhaft), ist es ein unentbehrliches Element im baltischen Puzzle. Es ist einer der beiden Nachbarn der Kaliningrader Oblast und besitzt mit Klaipeda einen der wichtigsten Ostseehäfen. Das zeigt deutlich, dass Litauen eine wesentliche geopolitische Rolle spielt. Mit Sicherheit ist sich die polnische Regierung dessen bewusst, wurde Litauen doch in den "Prioritäten der polnischen Außenpolitik 2012-16 «als einer der Schlüsselpartner in der Region aufgeführt. Es weist jedoch alles darauf hin, dass sich die polnische Regierung nach Jahren geduldigen Wartens auf eine Veränderung in den bilateralen Beziehungen entschieden hat, mit dem Fuß aufzustampfen und den Nachbarn zur Ordnung zu rufen.

\section{Neue polnische Ungeduld}

Eines der ersten Signale der Veränderungen in der polnischen Politik gegenüber Litauen war, dass sich der polnische Energiekonzern Polska Grupa Energetyczna (PGE) im Jahr 2011 aus der gemeinsamen Baufinanzierung eines Atomkraftwerks in Litauen zurückzog, was eines der wichtigsten geoökonomischen Projekte in diesem Teil Europas war. Ursprünglich war geplant worden, dass diese Investition von Litauen, Lettland, Estland und Polen gemeinsam finanziert würde. Erwähnenswert ist an dieser Stelle auch, dass auf der Baustelle eines Konkurrenzbaus in der Kaliningrader Oblast mit Hochdruck gearbeitet wird; polnische Journalisten werden dort in Ehren empfangen und auf dem Gelände herumgeführt. Die Russen hoffen, dass, wenn sich die Prognosen von Experten bestätigen, die Polen ab dem Jahr 2015 dauer- hafte Probleme wegen Strommangels gewärtigen werden und dieses Kraftwerk dann eine attraktive Stromquelle darstellen wird. Die Entscheidung der Polen bedeutete für die Litauer Schwierigkeiten - nach Abschalten des Kernkraftwerks in Ignalina (eine der Bedingungen für den Beitritt des Landes zur EU) blieben sie praktisch ohne eigene Energiequellen zurück und stehen vor dem realen Risiko, vollständig von russischen Quellen abhängig zu werden. Ignalina hatte 30 Prozent des Energiebedarfs des Landes abgedeckt und russisches Gas und Erdöl die restlichen 70 Prozent. Ein Jahr nach der Entscheidung Polens wurde dann das gesamte Projekt aufgegeben. In einem Referendum sprachen sich die Litauer gegen den AKWBau aus, und die neu gewählte Regierung teilt mit, dass sie sich dieser Entscheidung nicht entgegenstellen werde.

Die Entscheidung von PGE war mit Sicherheit eine politische Entscheidung, ähnlich wie der Kauf von Mozejki. Dabei klingen die Vorwürfe der litauischen Staatspräsidentin Dalia Grybauskaite, die Polen eines ausschließlich »kommerziellen« Denkens über Energiefragen tadelte, einerseits verständlich, andererseits aber wie das Lecken teilweise selbst vergossener Milch. Natürlich - es gibt keine Energie ohne Politik. Zugegeben werden muss aber auch, dass die politischen Signale aus Litauen nach Warschau in den letzten Jahren entmutigend waren, so als würde von polnischer Seite abermals eine Bedrohung ausgehen. Von den großen gemeinsamen Projekten sind also noch die erwähnte Rail Baltica und die Verbindung des Stromnetzes zwischen Polen und Litauen auf einer Länge von ungefähr $150 \mathrm{~km}$ aktuell. Falls die langjährigen Proteste der polnischen Bauern dies nicht blockieren, wird sie vom polnischen Ełk in die litauische Stadt Alytus führen.

\section{Die polnische Minderheit: umworbener Zankapfel}

Doch damit nicht genug: Seit zwei Jahren engagiert sich Polen energischer in Sachen polnischer Minderheit. Bisher mit wenig Erfolg - die interparlamentarische Gruppe, die für die Verhandlungen über das Bildungsgesetz verantwortlich ist, hat es nicht einmal geschafft, ein bescheidenes Memorandum auszuarbeiten. Dafür bekam sie vom damaligen litauischen Außenminister Audronius Ažubalis einen bezeichnenden Kommentar. Er sagte, dass Wilna keinen "älteren Bruder« brauche, und warf Polen Großmachtsgelüste nach russischem Muster vor.

Hoffnungen auf Veränderungen brachten die Parlamentswahlen in Litauen im vergangenen Jahr mit sich. Die Konservativen haben verloren und die Regierungsverantwortung übernahm ein Mitte-Links-Bündnis, dem auch die Wablaktion der Polen in Litauen (Akcja Wyborcza Polaków na Litwie) angehört. Diese hat im 
Parlament den Posten des stellvertretenden Vorsitzenden des Komitees für Menschenrechte erhalten, das Amt des Energieministers sowie vier Vizeministerposten: für Landwirtschaft, Transport, Bildung und Kultur. Die Regierung von Ministerpräsident Algirdas Butkevičius sendete auf diese Weise ein deutliches Signal, dass sie die Beziehungen zu Polen zu verbessern versuchen wird. Die erste versöhnliche Geste des neuen Außenministers Linas Linkevičius, der in Warschau für die Ablehnung des Gesetzes über die Originalschreibweise von Namen um Entschuldigung bat, wurde allerdings von Staatspräsidentin Grybauskaitè scharf kritisiert.

Die letzten Monate zeigen also, dass sich die polnisch-litauischen Beziehungen immer noch in einer Phase befinden, in der die kleinste Bewegung als Beleidigung des Nachbarn aufgefasst wird. So wurde auch eine Entscheidung der Selbstverwaltung in der polnischen Landgemeinde Puńsk behandelt, in der Litauer die Mehrheit stellen und deren Gemeindevorsteher ebenfalls Litauer ist. Sie will drei litauische Schulen schließen, aus denselben Gründen, aus denen in Litauen polnische Schulen geschlossen werden. 48 Schüler sollen in die Schulen von Sejny oder in das Städtchen Puńsk fahren. Auf die Entscheidung folgte eine blitzschnelle Reaktion litauischer Politiker: Staatspräsidentin Grybauskaite stellte fest, dass die "polnische Bildungspolitik für litauische Schulen nicht günstig« sei, der Ministerpräsident Litauens bot finanzielle Hilfe an und der litauische Bildungsminister fuhr nach Puńsk, um sich persönlich über die Angelegenheit zu informieren. Die Situation wird wiederum bis zur Unmöglichkeit dadurch verkompliziert, dass die Wablaktion der Polen in Litauen zur selben Zeit für die Litauer erstaunliche Korrekturen vorgeschlagen hat, nämlich die radikale Verschärfung des litauischen Abtreibungsgesetzes und die Einführung von obligatorischem Religionsunterricht in den litauischen Schulen, was auf Kritik des Erzbischofs von Kaunas, Sigitas Tamkevičius, stieß.

Die hier grob skizzierte Darstellung größerer oder kleinerer Konflikte beantwortet allerdings nicht die Frage nach der Hauptursache für die Abkühlung der polnisch-litauischen Beziehungen - falls es sie denn geben sollte. Die Polen haben die Geduld verloren, das ist uns nun bekannt. Warum aber hatten sich, noch bevor es dazu gekommen ist, die Litauer so hinter ihren Positionen verschanzt?

\section{Polnische Ambitionen, litauische Empfindlichkeiten}

Beim Versuch, die tieferen Gründe der Krise zu verstehen, mag die Analyse der bereits erwähnten baltischen Ambitionen Polens helfen. Außenminister Sikorski betreibt die Regionalpolitik so, dass Litauen das deutli- che Signal gegeben wird, umgangen zu werden. Die einzige deutliche Botschaft an unseren nördlichen Nachbarn in den letzten Monaten war eine ultimative Aussage zum geplanten Bau des Flüssiggasterminals in Klaipeda. Die Entscheidung der litauischen Regierung für diesen Terminal hat vielfältige Konsequenzen für die Region, darunter auch für Polen. Sie bedeutet, dass die Sinnhaftigkeit des Baus eines regionalen Terminals in Lettland mit einem Fragezeichen versehen wird. Weiterhin kann sie bedeuten, dass der Terminal in Swinemünde (Świnoujście) einen seiner regionalen Kooperationspartner verliert und in Klaipeda eine ernstzunehmende Konkurrenz gewinnt. Die Aussage von Sikorski, der von Litauen eine eindeutige Stellungnahme fordert - entweder der Terminal in Klaipeda oder die Gaspipeline Klaipeda-Warschau -, scheint jedoch kontraproduktiv zu sein und eher dazu zu dienen, die Stimmung anzuheizen, als sie zu beruhigen. Außerdem scheint hier nicht die grundlegende Tatsache beachtet zu werden, dass Litauen seinen Interessensvektor geändert hat, was führende Politiker in Wilna in den letzten Jahren mehrmals zum Ausdruck brachten. Polen bleibt für sie demnach natürlich ein wichtiger Partner bzw., wie es 2012 der damalige Ministerpräsident Andrius Kubilius elegant formuliert hat, ein »Transmissionsriemen« in den Westen. Die neue Doktrin sieht jedoch vor allem die Verstärkung der Kontakte mit Skandinavien vor. Skandinavien?! Die Überzeugung, dass Litauen partnerschaftliche Beziehungen mit Stockholm aufnehmen könnte, dass Stockholm für Wilna ein Gleichaltriger und nicht ein »älterer Bruder» sein könnte, scheint naiv oder verrückt zu sein.

Warum Stockholm? Ich sehe hier nur eine Antwort: Die Signale, die über die Ostsee gesendet werden, zeugen von dem Gefühl wachsender Vereinsamung und Marginalisierung Litauens und davon, dass es sich zunehmend zwischen den polnischen Hammer und den russischen Amboss gedrückt fühlt. Litauen sieht um sich herum keine Verbündeten, auch wenn es dies aus polnischer Sicht tun sollte. Litauen fürchtet sowohl die Dominanz Russlands als auch ein allzu starkes Polen, denn ein allzu starkes Polen ruft eindeutige Assoziationen hervor: die Besatzung in der Zwischenkriegszeit. Litauen macht uns den Vorwurf, dass wir mit Deutschland und Russland paktieren, und versucht, in die europäische Sonderklasse zu gelangen. Litauen will unabhängig sein, saugt aber gleichzeitig aufs Neue die russische Kultur auf. Und schließlich hat Litauen nur Feinde oder Verräter um sich herum, unter denen Polen den wichtigsten Platz einnimmt. Ob dies gerechtfertigte Überzeugungen sind, ist hier im Moment nicht wichtig. Wichtiger scheint die Antwort zu sein, was wir als Staat gemacht haben, um sie zu verhindern. Diese Antwort klingt nicht gut: Zuerst haben wir jahrelang geschwiegen, dann, als 
die Situation begann, kompliziert zu werden, haben wir mit dem Finger gedroht.

Ebenso wesentlich ist die Frage, ob man irgendetwas machen kann, um die Rückkehr alter Gespenster zu verhindern. Die Quellen der gegenseitigen Antipathie liegen tief in der Vergangenheit, sowohl der näheren als auch der ferneren. Die Litauer haben den Putsch mit Janajew im Gedächtnis, die Polen erinnern sich an litauische Faschisten. Die Litauer werden den Polen niemals die Inbesitznahme Wilnas durch General Lucjan Żeligowski im Oktober 1920 verzeihen, die Polen werden die Massenexekutionen von Polen mit Beteiligung von Litauern in Paneriai (poln.: Ponary) 1941 nicht vergessen. Im Gegensatz zur polnisch-deutschen Geschichte haben die Polen und die Litauer ihre tragischen historischen Ereignisse nicht aufgearbeitet. Es ist kein »Wir vergeben und bitten um Vergebung " gefallen, es treibt niemanden, einen ähnlich starken Satz auszusprechen. Stattdessen haben wir Kleinkrieg und Kräftemessen. Wilna ist die Arena für unglaublich absurde Kämpfe. Der Austausch der Treppenstufen in der Kapelle der Barmherzigen Muttergottes von Ostra Brama wächst zu einem internationalen Skandal an, für zweisprachige Informationstafeln in Bussen schrieben Beamte bis vor kurzem Mandate.

Bis auf weiteres scheint das polnische Rezept, um diesen gordischen Knoten zu lösen, klar: Entweder Ihr fangt an, in der Politik nach unseren Regeln zu spielen, oder Ihr verurteilt euch selbst zur Isolation. Das Problem liegt darin, dass eine solche Methode im Fall
Litauen nicht zum Erfolg führen kann. Davor warnte schon Michał Römer (1880-1945), der hervorragendste litauische Jurist und sensible Beobachter der polnischlitauischen Beziehungen der Zwischenkriegszeit, in seinem Tagebuch: "Die Vereinsamung, in die die polnische Politik Litauen drängte, ist für Litauen doppelt schwer. Einerseits wird es an den Scheideweg gestellt, aller Methoden beraubt, die eigene internationale Position mit Hilfe von Interessengemeinschaften zu stärken. Andererseits wird es im Streit mit Polen um Wilna schutz- und ratlos gemacht. Indem es von Polen aus den Beziehungen zu den Nachbarn ausgeschlossen wird von denen es träumte und in dessen Rahmen es seine Staatlichkeit konsolidieren wollte - kann Litauen aufgrund der geopolitischen Konstellation nicht gleichzeitig auf eigene Faust zwei anderen mächtigen Nachbarn entgegentreten, die zusammen Polens Rivalen sind: Deutschland und Russland. [...] Die Litauer sind leidenschaftlich, sie haben starke und tiefe Gefühle, sie haben Charakter. Polen sollte das wissen, denn das, was in seiner Geschichte am stärksten und am tiefsten war, das kam aus Litauen."

Trotz einer veränderten Landkarte ist es das Zitat wert, erinnert zu werden. Wenn die neue polnische Politik zur Verstärkung des Gefühls der Isolation in Litauen führen wird, dann wird die gegenwärtig kleine Spalte zwischen beiden Nationen wieder einmal zu einem Abgrund werden.

Übersetzung aus dem Polnischen: Silke Plate

\section{Über den Autor}

Michał Olszewski, war Leiter der Reportageabteilung der katholischen Wochenzeitung Tygodnik Powszechny und ist bis heute mit ihr verbunden. Gegenwärtig ist er Pressesprecher der Umweltorganisation Client Earth Poland. Er hat mehrere preisgekrönte Kurzgeschichtensammlungen verfasst, wie etwa Do Amsterdamu, sowie Essays und Kritiken, die in zahlreichen Literaturzeitschriften veröffentlicht wurden. 


\section{Außenminister Sikorski und Außenminister Linkevičius über die polnisch-litauische Zusammenarbeit}

\subsubsection{3}

Über die Vorbereitungen der litauischen EU-Ratspräsidentschaft, den Gipfel der Östlichen Partnerschaft in Wilna, die Angelegenheiten der Minderheit sowie die Intensivierung der Zusammenarbeit im Energiesektor sprachen in Warschau die Außenminister von Polen, Radosław Sikorski, und Litauen, Linas Linkevičius.

„Wir haben eine Sichtung der beiderseitigen Beziehungen vorgenommen, und ich habe konkrete Vorschläge zur Zusammenarbeit gemacht«, hielt Außenminister Sikorski fest. „Wir haben eine umfassende Unterstützung der ersten litauischen EU-Ratspräsidentschaft angeboten, der wir Erfolg wünschen. Wir haben ebenfalls organisatorische und politische Unterstützung für die Organisation des Gipfels der Östlichen Partnerschaft in Wilna angeboten, dessen Erfolg in unserem gemeinsamen Interesse liegt«, versicherte der Chef des polnischen Außenressorts. Außenminister Sikorski machte darüber hinaus das Angebot, Litauen an das polnische Gaspipeline-System anzuschließen, was allen baltischen Ländern ermöglichen würde, den zurzeit entstehenden Flüssiggasterminal in Swinemünde [Świnoujście] zu nutzen.

"Wir sind nach Polen gekommen, um einen sehr wichtigen Nachbarn und Verbündeten zu besuchen«, sagte Außenminister Linas Linkevičius. »Vor uns liegen so viele gemeinsame Projekte, dass die Missverständnisse und Unklarheiten der Vergangenheit angehören sollten«, fügte er hinzu.

Die Minister sprachen auch über die Rechte der polnischen Minderheit in Litauen. Außenminister Sikorski unterstrich, dass wir gemeinsam mit den internationalen Organisationen auf die Verabschiedung des von der litauischen Regierung versprochenen Minderheiten-Gesetzes warten, das das angemessenste Format zu sein scheint, um die Forderungen nationaler Minderheiten zu regeln. »Ich sehe keinen Grund, warum das Gesetz über die nationalen Minderheiten, das bis zum Jahr 2010 galt, nicht weiter gelten sollte«, fügte der litauische Außenminister bezugnehmend auf die Frage der Rechte der polnischen Minderheit in Litauen hinzu.

Am Ende des Treffens äußerte der polnische Außenminister die Hoffnung, dass die heutigen Gespräche und die Gespräche der Ministerpräsidenten am 12. Februar einen konkreten Handlungsplan für Polen und Litauen - den Nachbarn und Ländern, die sich nahe ständen, - zeitigen werden. „Ich hoffe, dass unsere Gespräche es der litauischen Seite erleichtern, ihren Standpunkt so vorzubereiten, dass sich unsere Beziehungen verbessern können«, fasste Außenminister Sikorski zusammen.

Das Büro des Pressesprechers

Außenministerium

Übersetzung aus dem Polnischen: Silke Plate

Quelle: Ministerstwo Spraw Zagranicznych RP [Außenministerium der Republik Polen] http://www.msz.gov.pl/pl/p/msz_pl/polityka_zagra niczna/europa/partnerstwo_wschodnie/ministrowie_sikorski_i_linkevicius_o_wspolpracy_polsko_litewskiej (abgerufen am 18.03.2013)

\section{Außenministerium: Neue Dynamik in den Beziehungen zu Litauen nach der Verabschiedung des Namensgesetzes}

\section{PAP* vom 12.02.2013}

Außenminister Radosław Sikorski sagte am Dienstag, dass man von einer neuen Dynamik in den polnisch-litauischen Beziehungen nach der Verabschiedung des Gesetzes über die Schreibung der polnischen Namen durch das litauische Parlament sprechen könne. Am Dienstag wird Ministerpräsident Algirdas Butkevičius zu einem Besuch nach Warschau kommen.

Sikorski erinnerte im 1. Programm des Polnischen Radio daran, dass er in seiner Rede über die Prioritäten der Außenpolitik im Sejm im vergangenen Jahr gesagt hatte, dass wir auf einen Neuanfang in den Beziehungen zu Litauen und seiner neuen Regierung warten.

"Dies findet nun statt«, sagte der Minister und fügte hinzu, dass der Besuch des litauischen Außenministers, Linas Linkevičius, in der vergangenen Woche gut verlaufen sei und gemeinsam die Gespräche der Regierungschefs vorbereitet worden seien.

„Dass der Außenminister Litauens dafür um Verzeihung bat, dass die litauischen Parlamentarier am Tag des letzten Besuches von Lech Kaczyński einen guten Kompromiss für einen Gesetzesentwurf über die Schreibweise von 
Namen abgelehnt hatten, ist erstens eine schöne Geste und führt zweitens zu der logischen politischen Schlussfolgerung, dass dieser Fehler korrigiert werden muss«, sagte der Minister. "Wenn der Fehler korrigiert werden wird, d. h. dass beispielsweise dasselbe Gesetz noch einmal und dieses Mal erfolgreich verabschiedet wird, wird man von einer neuen Dynamik in den polnisch-litauischen Beziehungen sprechen können, an denen uns liegt«, erklärte der Minister.

In der vergangenen Woche hatte der Chef des litauischen Außenministeriums, Linas Linkevičius, während seines Besuchs in Polen im Gespräch mit der Tageszeitung "Rzeczpospolita " gesagt, dass es ihm leid tue, dass das Parlament während des letzten Besuchs von Staatspräsident Lech Kaczyński in Wilna (2010) das Gesetz über die Schreibweise der polnischen Namen abgelehnt hatte. "Ich war damals kein Abgeordneter, aber ich möchte mich dafür entschuldigen«, sagte der litauische Außenminister.

Sikorski erinnerte am Dienstag daran, dass Litauen in der zweiten Jahreshälfte die Arbeiten der EU führen wird und es darüber hinaus in unserem gemeinsamen Interesse liegt, dass der Gipfel der Östlichen Partnerschaft, der im November in Wilna stattfinden wird, erfolgreich durchgeführt wird.

"Wir möchten, dass die Länder der Östlichen Partnerschaft Assoziierungsabkommen mit der EU unterzeichnen«, sagte der Außenminister.

Die Hauptthemen beim Besuchs des litauischen Ministerpräsidenten am Dienstag in Warschau sollen die wirtschaftliche Zusammenarbeit, die Umsetzung der internationalen Projekte Rail Baltica, Via Baltica, die Energiepolitik sowie die Probleme der litauischen Polen sein.

*Polnische Presseagentur

Übersetzung aus dem Polnischen: Silke Plate

Quelle: Ministerstwo Spraw Zagranicznych RP [Außenministerium der Republik Polen] http://www.msz.gov.pl/pl/p/p/msz_pl/szu kaj?search=true\&searchCategory=\&searchWord=Litwa (abgerufen am 14.03.2013).

\section{Sikorski: Gaspipeline in die baltischen Länder gegen die Isolation der Region PAP* vom 13.02.2013}

"Die Zusammenarbeit Polens und der baltischen Staaten im Bereich des Gastransports kann zur Aufhebung der Isolation der Staaten dieser Region im Energiesektor beitragen«, sagte Außenminister Radosław Sikorski am Mittwoch in Riga. Er betonte, dass der Erfolg des Projekts von Litauen abhänge.

Außenminister Sikorski unternimmt einen eintägigen offiziellen Besuch in Lettland, wo er sich mit seinem lettischen Amtskollegen Edgars Rinkēvičs, Parlamentspräsidentin Solvita Aboltina und Ministerpräsident Valdis Dombrovskis traf.

Nach dem Treffen mit Dombrovskis unterstrich Sikorski, dass das Projekt der Zusammenarbeit im Bereich Gastransport mit einer Zufinanzierung der EU - aus den Mitteln der der Europäischen Kommission zur Verfügung stehenden Fazilität Connecting Europe - bis zu einer Höhe von 70 Prozent, abgesehen von unseren länderspezifisch garantierten Geldern, rechnen kann.

»Estland hat umfangreiche Erdgasspeicher, wir werden in Kürze einen Flüssiggasterminal haben und Möglichkeiten, Gaspipelines in die baltischen Länder zu legen«, sagte Sikorski Journalisten. Polen baut einen Flüssiggasterminal in Swinemünde [Świnoujście], der 2014 in Betrieb genommen werden soll. Sikorski hebt hervor, dass für den Erfolg des Projekts der Standpunkt Litauens eine große Bedeutung hat. „Die Entscheidung liegt bei Litauen, ob es sich anschließen will oder ob es bevorzugt, seinen eigenen Flüssiggasterminal zu bauen. Aus unseren Kalkulationen geht hervor, dass man nicht dafür sorgen kann, dass beide Investitionen rentabel sind«, sagte Sikorski.

Am Dienstag hatten die Ministerpräsidenten Donald Tusk und Algirdas Butkevičius bekannt gegeben, dass Polen und Litauen Schlüsselprojekte im Bereich Energie, Verbindungen der Strom- und der Gasnetze, fortsetzen werden.

Gleichzeitig erinnerte Butkevičius daran, dass Litauen den politischen Beschluss über eine vollständige Umsetzung des Dritten Liberalisierungspakets für den Europäischen Energiemarkt gefasst hat, was bis Ende 2014 stattgefunden haben soll. Er hebt hervor, dass bis zu diesem Zeitpunkt in Klaipeda ein Flüssiggasterminal entstehen soll, was eine Diversifizierung des Gassektors in Litauen bedeuten wird. Er fügte hinzu, dass Litauen bereits Verträge für die Lieferung von Flüssiggas aushandelt.

*Polnische Presseagentur

Übersetzung aus dem Polnischen: Silke Plate

Quelle: Ministerstwo Spraw Zagranicznych RP [Außenministerium der Republik Polen] http://www.msz.gov.pl/pl/p/msz_pl/aktualnosci/msz_w_ mediach/sikorski__gazociag_do_krajow_baltyckich_przeciw_izolacji_regionu__depesza_z_13_02_2013 (abgerufen am 18.03.2013). 


\section{Sympathie gegenüber anderen Nationen}

Tabelle 1: Veränderungen der Sympathie gegenüber ausgewählten Nationen (\%)

\begin{tabular}{|c|c|c|c|c|c|c|c|c|c|c|c|c|c|c|c|c|c|c|c|}
\hline & W & $\underset{\downarrow}{\varpi}$ & చ్ర & $\begin{array}{l}\text { ஏర } \\
\text { }\end{array}$ & $\underset{\text { అ }}{ }$ & $\underset{\infty}{\circ}$ & $\underset{ర}{\varpi}$ & 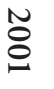 & $\begin{array}{l}\text { No } \\
\text { O }\end{array}$ & No & $\begin{array}{l}\text { No } \\
\text { O }\end{array}$ & $\begin{array}{l}\text { No } \\
\text { O }\end{array}$ & $\begin{array}{l}\text { No } \\
\text { ᄋ̆ }\end{array}$ & 용 & 응 & 응 & 苍 & 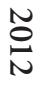 & $\underset{\omega}{\sim}$ \\
\hline $\begin{array}{l}\text { Ame- } \\
\text { rikaner }\end{array}$ & 62 & 58 & 63 & 59 & 64 & 61 & 54 & 50 & 58 & 56 & 45 & 46 & 49 & 44 & 47 & 45 & 43 & 51 & 43 \\
\hline Belarussen & 19 & 17 & 18 & 21 & 22 & 19 & 18 & 26 & 26 & 22 & 21 & 18 & 23 & 27 & 34 & 34 & 31 & 33 & 30 \\
\hline Briten & 47 & 41 & 51 & 51 & 55 & 50 & 45 & 44 & 51 & 49 & 46 & 50 & 50 & 53 & 51 & 50 & 44 & 54 & 47 \\
\hline Bulgaren & 19 & 16 & 23 & 23 & 23 & 20 & 20 & 25 & 26 & 24 & 22 & 19 & 24 & 29 & 35 & 37 & 33 & 38 & 32 \\
\hline Chinesen & - & - & - & - & - & 21 & 19 & 22 & 26 & 22 & 20 & 16 & 18 & 22 & 31 & 29 & 31 & 32 & 27 \\
\hline Deutsche & 23 & 26 & 35 & 43 & 38 & 32 & 31 & 32 & 38 & 36 & 33 & 34 & 33 & 30 & 38 & 39 & 38 & 43 & 38 \\
\hline Franzosen & 61 & 51 & 67 & 62 & 60 & 58 & 53 & 55 & 51 & 48 & 45 & 43 & 48 & 52 & 49 & 51 & 45 & 53 & 41 \\
\hline Georgier & - & - & - & - & - & - & - & - & - & - & - & - & - & 27 & 35 & 32 & 34 & 37 & 31 \\
\hline Griechen & - & - & - & - & - & - & - & 43 & 44 & 45 & 44 & 41 & 47 & 49 & 46 & 47 & 43 & 40 & 35 \\
\hline Iren & - & - & - & - & - & - & - & - & - & - & 39 & 43 & 49 & 54 & 47 & 46 & 40 & 47 & 44 \\
\hline Italie & 63 & 54 & 66 & 62 & 63 & 55 & 53 & 54 & 54 & 53 & 50 & 52 & 52 & 51 & 54 & 52 & 47 & 55 & 46 \\
\hline Juden & 15 & 17 & 25 & 26 & 28 & 19 & 19 & 19 & 23 & 21 & 18 & 20 & 20 & 23 & 34 & 31 & 31 & 33 & 28 \\
\hline Kroaten & - & - & - & - & - & - & - & 25 & 29 & 28 & - & - & - & - & - & 40 & 36 & 44 & 37 \\
\hline Litauer & 24 & 22 & 35 & 36 & 36 & 29 & 30 & 36 & 38 & 36 & 38 & 32 & 36 & 38 & 41 & 42 & 36 & 40 & 32 \\
\hline $\begin{array}{l}\text { Nieder- } \\
\text { länder }\end{array}$ & - & - & - & - & - & - & - & - & - & - & 42 & 42 & 48 & 48 & 46 & 46 & 45 & 49 & 42 \\
\hline Norweger & - & - & - & - & - & - & - & - & - & - & - & - & - & - & 46 & 46 & 42 & 52 & 43 \\
\hline $\begin{array}{l}\text { Öster- } \\
\text { reicher }\end{array}$ & 41 & 37 & 48 & 49 & 47 & 43 & 40 & 37 & 40 & 36 & 31 & 30 & 36 & 36 & 43 & 43 & 39 & 48 & 40 \\
\hline Rumäne & 9 & 8 & 11 & 12 & 11 & 10 & 13 & 15 & 15 & 15 & 14 & 12 & 16 & 16 & 2 & 26 & 24 & 26 & 21 \\
\hline Russen & 17 & 16 & 17 & 21 & 20 & 19 & 17 & 23 & 24 & 22 & 18 & 16 & 22 & 24 & 30 & 34 & 32 & 34 & 31 \\
\hline Schweden & 44 & 40 & 53 & 52 & 49 & 43 & 41 & 44 & 46 & 48 & 42 & 39 & 43 & 44 & 45 & 44 & 41 & 51 & 42 \\
\hline Serben & 10 & 12 & 14 & 15 & 16 & 13 & 14 & 17 & 18 & 17 & 15 & 12 & 18 & 19 & 29 & 31 & 28 & 31 & 27 \\
\hline Slowakeı & 33 & 32 & 44 & 38 & 39 & 36 & 34 & 42 & 42 & 43 & 41 & 36 & 44 & 48 & 51 & 51 & 49 & 57 & 48 \\
\hline $\begin{array}{l}\text { Sinti und } \\
\text { Roma }\end{array}$ & - & 6 & 10 & 12 & 10 & 10 & 13 & 17 & 15 & 16 & 15 & 13 & 14 & 14 & 21 & 23 & 22 & 24 & 20 \\
\hline Spanier & - & - & - & - & - & - & - & - & - & - & 47 & 47 & 53 & 49 & 52 & 51 & 47 & 53 & 45 \\
\hline Tschechen & 38 & 30 & 43 & 44 & 45 & 41 & 44 & 47 & 50 & 50 & 49 & 46 & 52 & 53 & 53 & 53 & 51 & 58 & 51 \\
\hline Türken & - & - & - & - & - & - & - & - & - & - & - & 14 & 17 & 18 & 28 & 28 & 29 & 28 & 24 \\
\hline Ukrainer & 12 & 9 & 14 & 16 & 15 & 13 & 16 & 19 & 22 & 19 & 29 & 23 & 24 & 25 & 34 & 34 & 32 & 32 & 31 \\
\hline Ungarn & 47 & 41 & 56 & 48 & 47 & 44 & 44 & 47 & 48 & 45 & 43 & 39 & 45 & 45 & 48 & 49 & 46 & 52 & 42 \\
\hline $\begin{array}{l}\text { Viet- } \\
\text { namesen }\end{array}$ & - & - & - & - & - & 20 & 21 & 23 & 24 & 19 & 17 & 15 & 18 & 18 & 28 & 29 & 30 & 29 & 25 \\
\hline
\end{tabular}

Anm.: Juden sowie Sinti und Roma werden als Nationen aufgefasst.

Quelle: CBOS BS/12/2013 Stosunek Polaków do innych narodów [Das Verbältnis der Polen zu anderen Nationen]. Warszawa 02/2013. www.cbos.pl 


\section{Vom 5. bis zum 18. März 2013}

\begin{tabular}{|c|c|}
\hline 05.03 .2013 & $\begin{array}{l}\text { In einer Sondersitzung der Fraktion von Recht und Gerechtigkeit (Prawo i Sprawiedliwość - PiS) stellt Piotr } \\
\text { Gliński, Schattenkandidat von PiS für das Amt des Ministerpräsidenten, das Programm seines Schattenkabi- } \\
\text { netts vor. In der gegenwärtigen Krise Polens sei eine Technokratenregierung die richtige Lösung, um die viel- } \\
\text { schichtigen Probleme in Bereichen wie Wirtschaft, Finanzen, Demographie anzugehen. Da eine solche Exper- } \\
\text { tenregierung nicht in der Politik verwurzelt sei, könne sie das verlorene Vertrauen der Bevölkerung in die Poli- } \\
\text { tik und in eine Regierung wiederaufbauen. Gliński behauptet, dass er von der Mehrheit der Medien boykot- } \\
\text { tiert werde, was nicht den Regeln einer Demokratie entspreche. }\end{array}$ \\
\hline 06.03 .2013 & $\begin{array}{l}\text { In Warschau findet ein Treffen der Regierungschefs der Visegrád-Gruppe (Polen, Slowakei, Tschechien, Ungarn) } \\
\text { statt, an dem auch Bundeskanzlerin Angela Merkel und der französische Staatspräsident François Hollande teil- } \\
\text { nehmen. Auf der Pressekonferenz hebt Ministerpräsident Donald Tusk hervor, dass sich die anwesenden Länder } \\
\text { für die Einheit Europas und die Zukunft der europäischen Integration verantwortlich fühlen. Die europäische } \\
\text { Einheit werde mit Hilfe der Währungs- und Wirtschaftsunion, der Wettbewerbsfähigkeit der EU-Länder und } \\
\text { der Stärkung der europäischen Verteidigungsfähigkeit erreicht. Tusk weist auf die Absichtserklärung der Ver- } \\
\text { teidigungsminister der Visegrád-Gruppe über die Einberufung einer gemeinsamen militärischen Einheit hin, } \\
\text { die Teil der EU-Verteidigungsidentität werden soll. Polen hat zurzeit den Vorsitz der Visegrád-Gruppe inne. }\end{array}$ \\
\hline 07.03 & $\begin{array}{l}\text { In einem Radiointerview bekräftigt Ryszard Kalisz, Demokratische Linksallianz (Sojusz Lewicy Demokratycz- } \\
\text { nej-SLD), dass er nicht beabsichtige, aus der SLD auszutreten. Am Vortag hatte der SLD-Vorstand Kalisz für } \\
\text { maximal drei Monate die Rechte als SLD-Mitglied aberkannt. Hintergrund ist sein Engagement für die politi- } \\
\text { sche Bewegung "Europa Plus«, die SLD-Chef Leszek Miller als Konkurrenz für die SLD betrachtet und abge- } \\
\text { lehnt hatte. }\end{array}$ \\
\hline 08.0 & $\begin{array}{l}\text { Im Sejm wird der Antrag von Recht und Gerechtigkeit (Prawo i Sprawiedliwość-PiS) auf ein konstruktives Miss- } \\
\text { trauensvotum gegen die Regierung von Ministerpräsident Donald Tusk (Bürgerplattform/Platforma Obywatel- } \\
\text { ska - PO) mit } 236 \text { Gegenstimmen abgelehnt. } 137 \text { Abgeordnete stimmen dafür, } 41 \text { enthalten sich der Stimme. }\end{array}$ \\
\hline 09.03 .2013 & $\begin{array}{l}\text { In Warschau findet ein Parteitreffen der Demokratischen Linksallianz (Sojusz Lewicy Demokratycznej-SLD) } \\
\text { zur Situation des Gesundheitswesens und der Verantwortung des Staates statt. Der Parteivorsitzende Leszek } \\
\text { Miller wirft der Regierung vor, sich nicht ausreichend um das Gesundheitswesen zu kümmern. Der Gesundheits- } \\
\text { beauftragte der SLD, Marek Balicki, verweist auf die wachsende Unzufriedenheit der Bürger mit dem Gesund- } \\
\text { heitssystem. Die derzeitige Privatisierung sei ein falscher Schritt. Darüber hinaus sei der Mangel an Ärzten und } \\
\text { Krankenschwestern ein zunehmendes Problem in Polen. }\end{array}$ \\
\hline 10.03 .2013 & $\begin{array}{l}\text { Bei einem Besuch des stellvertretenden Sejmmarschall Eugeniusz Greszczak in Wilna (Litauen) aus Anlass des } \\
\text { Jahrestages der Unabhängigkeit (1990) teilt der stellvertretende Präsident des litauischen Parlaments, Gediminas } \\
\text { Kirkilas, mit, dass die Litauisch-Polnische Abgeordnetenversammlung ihre Tätigkeit wieder aufnehmen wird. } \\
\text { Hintergrund für die Unterbrechung waren Probleme im Bereich der Rechte für die polnische Minderheit in } \\
\text { Litauen. }\end{array}$ \\
\hline 11.03. & $\begin{array}{l}\text { Bei der Eröffnung der moldawisch-polnischen Parlamentsversammlung in der moldawischen Hauptstadt } \\
\text { Chişinău unterstreicht Senatsmarschall Bogdan Borusewicz, dass Moldawien ein Land mit einer fundierten } \\
\text { Demokratie sei und von der EU zurzeit als beispielhaft beurteilt wird. Eine anhaltende politische Krise in Mol- } \\
\text { dawien wäre nachteilig für die Assoziierungsverhandlungen mit der EU. Polen setze sich bei den Ländern der } \\
\text { „Östlichen Partnerschaft« dafür ein, dass der Gipfel der Östlichen Partnerschaft in Wilna (Litauen) im Novem- } \\
\text { ber für Moldawien ein deutlicher Schritt in Richtung EU werde. Der moldawische Parlamentspräsident Marian } \\
\text { Lupu betont den strategischen Charakter der Beziehungen zu Polen. Polen sei das einzige Land, mit dem eine } \\
\text { institutionalisierte Zusammenarbeit der Parlamente besteht. }\end{array}$ \\
\hline 12.03 .2013 & $\begin{array}{l}\text { Ministerpräsident Donald Tusk trifft sich mit den Ministern des Wirtschafts-, des Schatz- und des Innenres- } \\
\text { sorts sowie dem Leiter der Kanzlei beim Ministerpräsidenten und dem Chef des polnischen Energiekonzerns } \\
\text { PGE. Thematisiert werden strategische Fragen der Energiepolitik. Tusk hebt hervor, dass unabhängig von der } \\
\text { Errichtung eines Atomkraftwerks in Polen und der Energiegewinnung aus Schiefergas oder auf der Basis erneu- } \\
\text { erbarer Energiequellen Braunkohle für Polen weiterhin eine Schlüsselbedeutung haben werde. }\end{array}$ \\
\hline 13.03 .2013 & $\begin{array}{l}\text { Auf einer Sitzung des Senatsausschusses für die nationale Wirtschaft teilt der stellvertretende Schatzminister } \\
\text { Rafał Baniak mit, dass für das Jahr } 2013 \text { mit Einnahmen in Höhe von zirka } 5 \text { Mrd. Zloty aus der Privatisie- } \\
\text { rung von Teilen des Staatsschatzes gerechnet wird. }\end{array}$ \\
\hline 14.03 .2013 & $\begin{array}{l}\text { Sportministerin Joanna Mucha verteidigt die Auszahlung von Prämien in Millionenhöhe an die Manager der } \\
\text { Fußballeuropameisterschaft EURO 2012. Die Verträge seien mit ihren Amtsvorgängern unterzeichnet worden, } \\
\text { daher sei eine Nichtauszahlung ein Rechtsbruch. Die Opposition hatte scharfe Kritik an den Prämien geäußert. }\end{array}$ \\
\hline
\end{tabular}




\begin{tabular}{|l|l|}
\hline 15.03.2013 & $\begin{array}{l}\text { Fiat Auto Poland in Tychy (Woiwodschaft Schlesien/woj. śląskie) nimmt die letzte Gruppenentlassung vor. Von } \\
\text { der Massenentlassung, die über drei Monate verteilt wurde, sind 1.450 Arbeitnehmer betroffen. Wirtschaftsmi- } \\
\text { nister Janusz Piechociński hatte sich in die Verhandlungen eingeschaltet. }\end{array}$ \\
\hline 16.03.2013 & $\begin{array}{l}\text { Im historischen Konferenzsaal Sala BHP in Danzig (Gdańsk) versammeln sich zirka 100 Organisationen, Ver- } \\
\text { eine und Gewerkschaften aus unterschiedlichen Bereichen unter dem Namen Plattform der Empörten (Plat- } \\
\text { forma Oburzonych). Das Treffen wurde vom Vorsitzenden der Gewerkschaft Solidarność (NSZZ Solidarność), } \\
\text { Piotr Duda, organisiert. Duda fordert mehr direkte Demokratie und eine entsprechende Überarbeitung des } \\
\text { Gesetzes über Volksentscheide. }\end{array}$ \\
\hline 17.03.2013 18.03.2013 & $\begin{array}{l}\text { In Leipzig endet die Leipziger Buchmesse, deren Programmreihe »tranzyt« den Schwerpunkt auf Literatur aus } \\
\text { Polen, der Ukraine und Belarus legte. }\end{array}$ \\
\hline $\begin{array}{l}\text { Nach neuesten Angaben des Statistischen Hauptamts (Główny Urząd Statystyczny - GUS) betrug der durch- } \\
\text { schnittliche Bruttolohn im Unternehmensbereich (mehr als neun Arbeitnehmer) im Februar 2013 3.710 Zloty } \\
\text { (zirka 895 Euro). Dies ist eine Steigerung von 0,8 \% im Vergleich zu Januar 2013 und von 4 \% im Vergleich } \\
\text { zu Februar 2012. }\end{array}$ \\
\hline
\end{tabular}

Sie können die gesamte Chronik seit 2007 auch auf http://www.laender-analysen.de/polen/ unter dem Link»Chronik« lesen. 
Die Polen-Analysen erscheinen zweimal monatlich als E-Mail-Dienst. Sie werden gemeinsam vom Deutschen PolenInstitut Darmstadt, der Bremer Forschungsstelle Osteuropa und der Deutschen Gesellschaft für Osteuropakunde in Partnerschaft mit dem Willy Brandt Zentrum für Deutschland- und Europastudien an der Universität Wroclaw (Breslau) herausgegeben.

Ein Archiv der Polen-Analysen finden Sie im Internet unter www.laender-analysen.de/polen

Kostenloses Abonnement unter http://www.deutsches-polen-institut.de/Newsletter/subscribe.php

Diese Analysen finden Sie online als Lizenzausgabe auf bpb.de

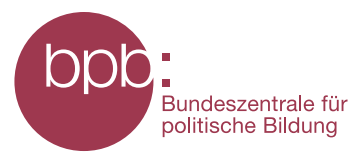

\section{Deutsches Polen-Institut Darmstadt}

Das Deutsche Polen-Institut Darmstadt (DPI) ist ein Forschungs-, Informations-, und Veranstaltungszentrum für polnische Kultur, Geschichte, Politik, Gesellschaft und die deutsch-polnischen Beziehungen, die sich im Kontext der europäischen Integration entwickeln. Das seit März 1980 aktive und bis 1997 von Gründungsdirektor Karl Dedecius geleitete Institut ist eine Gemeinschaftsgründung der Stadt Darmstadt, der Länder Hessen und Rheinland-Pfalz sowie des Bundes. 1987 wurden die Kultusminister der Länder und 2011 das Auswärtige Amt weitere institutionelle Träger. Einen wesentlichen Beitrag zur Verwirklichung der Institutsziele leisten private Stiftungen. Das DPI hat satzungsgemäß die Aufgabe, durch seine Arbeit zur Vertiefung der gegenseitigen Kenntnisse des kulturellen, geistigen und gesellschaftlichen Lebens von Polen und Deutschen beizutragen.

Ziel der Vermittlertätigkeit des DPI ist es, „die zu interessieren, auf die es politisch, wirtschaftlich, gesellschaftlich und kulturell im deutsch-polnischen Verhältnis ankommt (Leitlinien 1997). Es geht um die Entscheider und Multiplikatoren in Politik, Kultur, Bildung, Verwaltung, Medien und Wirtschaft und, wesentlich stärker ausgeprägt als bisher, um das Hineinwirken in Wissenschaft, Forschung und Bildung.

Derzeit bemüht sich das DPI in Kooperation mit den verstreuten Orten wissenschaftlicher Polen-Kompetenz an deutschen Hochschulen und Forschungsinstituten verstärkt darum, ausgehend von einer Bestandsaufnahme deutscher Polen-Forschung Ort wissenschaftlicher Forschung und verbindendes, vernetzendes und kooperierendes Zentrum zu werden. Ausgangspunkt der Neuausrichtung ist die kaum mehr kontrollierbare Dynamik des Rückbaus der Ressourcen der wissenschaftlichen Polen-Kompetenz in den unterschiedlichen Disziplinen. Mit der über 60.000 Bände zählenden multidisziplinären Fachbibliothek für Polen, die eine einzigartige Sammlung polnischer Literatur in der Originalsprache und in deutscher Übersetzung umfasst, ist das DPI bereits ein geschätzter Ort der Recherche und des wissenschaftlichen Arbeitens. (www.deutsches-polen-institut.de)

\section{Forschungsstelle Osteuropa an der Universität Bremen (www.forschungsstelle.uni-bremen.de)}

1982 gegründet, widmet sich die Forschungsstelle Osteuropa an der Universität Bremen der interdisziplinären Analyse der Länder Ost- und Ostmitteleuropas in Zeitgeschichte und Gegenwart. Der Forschungsschwerpunkt liegt dabei auf der Rolle von »Dissens und Konsens«, von Opposition und Zivilgesellschaft in ihrem historischen, politischen, gesellschaftlichen und kulturellen Kontext. Die Forschungsstelle besitzt in ihrem Archiv eine einzigartige Sammlung alternativer Kulturgüter und unabhängiger Texte aus den ehemaligen sozialistischen Ländern. Darunter befindet sich auch eine umfangreiche Sammlung des "Zweiten Umlaufs«, die das Schrifttum und Dokumente unabhängiger Initiativen und gesellschaftlicher Gruppen in Polen aus der Zeit von 1976 bis zum Umbruch umfasst. Hinzu kommt eine umfangreiche Bibliothek mit wissenschaftlicher Literatur. Mit Archiv, Bibliothek und zwei wissenschaftlichen Abteilungen ist die Forschungsstelle auch eine Anlaufstelle sowohl für Gastwissenschaftler als auch für die interessierte Öffentlichkeit.

Eine der Hauptaufgaben der Forschungsstelle ist die Information der interessierten Öffentlichkeit. Dazu gehören unter anderem regelmäßige E-Mail-Informationsdienste für Politik, Wirtschaft, Zivilgesellschaft und Medien.

\section{Herausgegeben mit finanzieller Unterstützung der Stiftung für deutsch-polnische Zusammenarbeit}

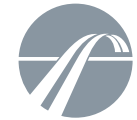

FUNDACJA WSPÓEPRACY

STIFTUNG

STIFTUNG

FUR DEUTSCH-POLNISCHE
ZUSAMMENARBEIT

Die Meinungen, die in den Polen-Analysen geäußert werden, geben ausschließlich die Auffassung der Autoren wieder.

Abdruck und sonstige publizistische Nutzung sind nach Rücksprache mit der Redaktion gestattet.

Redaktion: Prof. Dr. Dieter Bingen (verantwortlich) (Darmstadt), Silke Plate, M.A. (Bremen) Technische Gestaltung: Matthias Neumann

Polen-Analysen-Layout: Cengiz Kibaroglu, Matthias Neumann

Alle Ausgaben der Polen-Analysen sind mit Themen- und Autorenindex archiviert unter www.laender-analysen.de

Die Polen-Analysen werden im Rahmen der Datenbank World Affairs Online (WAO) ausgewertet und sind im Portal IREON www.ireon-portal.de recherchierbar.

ISSN 1863-9712 @ 2013 by Deutsches Polen-Institut Darmstadt und Forschungsstelle Osteuropa, Bremen

Kontakt: Dr. Andrzej Kaluza, Presse- und Öffentlichkeitsarbeit, Deutsches Polen-Institut, Mathildenhöhweg 2,

D-64287 Darmstadt, Tel.: 06151/4985-13, Fax: 06151/4985-10, E-Mail: polen-analysen@dpi-da.de, Internet: www.laender-analysen.de/polen 